\title{
Multiple diagnostic tests demonstrate an increased risk of canine heartworm disease in northern Queensland, Australia
}

Jessica L. Panetta, Nichola Eliza Davies Calvani®i, Bronwyn Orr, Aldo Gianfranco Nicoletti, Michael P. Ward (1) and Jan Šlapeta* ${ }^{*}$

\begin{abstract}
Background: Canine heartworm (Dirofilaria immitis) is a life-threatening infection of dogs with a global distribution. Information on the prevalence of D. immitis and associated risk factors for canine heartworm antigen positivity —and thus disease - in Australia is scarce or outdated. The current reference method for D. immitis diagnosis in dogs is via the detection of heartworm antigen in blood using commercially available microwell-based enzyme-linked immunosorbent assays (ELISAs). Heat treatment of canine plasma prior to testing has been suggested to increase test sensitivity. The aim of the current study was to estimate the prevalence of D. immitis in dogs confined to shelters in Queensland, Australia. The impact of heat treatment on antigen test results was also assessed.
\end{abstract}

Methods: Blood samples $(n=166)$ were collected directly from dogs in seven shelters across Queensland (latitudinal span of approx. $1700 \mathrm{~km}$ ) into EDTA blood collection tubes. A commercially available ELISA (DiroCHEK ${ }^{\circledR}$ ) was used to detect canine heartworm antigen in untreated and heat-treated plasma. Whole blood was concurrently tested for the presence of microfilariae and D. immitis DNA using a modified Knott's test and real-time PCR, respectively. Risk factors (age, gender, source, location) associated with the odds of positivity for canine heartworm were assessed using binary logistic regression models.

Results: A total of 16 dogs (9.6\%; 95\% confidence interval [Cl]: 5.9-15.2\%) were positive for canine heartworm based on combined test results. Heat treatment did not impact on the positivity of $D$. immitis antigen within samples (Cohen's kappa $=0.98$ ), but the optical density was significantly increased in paired plasma samples for $D$. immitis antigen-positive samples (Wilcoxon matched-pairs signed rank test, two-tailed $P<0.01$ ). Location of the dog in a shelter in northern Queensland was the only risk factor significantly associated with the odds of a dog being more likely to be D. immitis antigen positive (odds ratio: $4.39 ; 95 \%$ Cl: 1.26-13.51). All samples positive for the modified Knott's test were also positive for D. immitis DNA by PCR.

Conclusions: This study demonstrated the presence of heartworm-positive dogs in shelters in Queensland, with positive animals significantly more likely to occur in northern Queensland than southern Queensland. Sustained testing for the presence of $D$. immitis microfilariae and antigen remain important diagnostic tools in areas with known and re-emerging canine heartworm activity.

Keywords: Dirofilaria immitis, Prevalence, Microfilariae, Shelter dogs, Antigen test, Heat-treatment, Knott's test, PCR, Queensland, Australia

*Correspondence: jan.slapeta@sydney.edu.au

Sydney School of Veterinary Science, Faculty of Science, The University

of Sydney, Sydney, NSW 2006, Australia original author(s) and the source, provide a link to the Creative Commons licence, and indicate if changes were made. The images or other third party material in this article are included in the article's Creative Commons licence, unless indicated otherwise in a credit line to the material. If material is not included in the article's Creative Commons licence and your intended use is not permitted by statutory regulation or exceeds the permitted use, you will need to obtain permission directly from the copyright holder. To view a copy of this licence, visit http://creativecommons.org/licenses/by/4.0/. The Creative Commons Public Domain Dedication waiver (http://creativeco mmons.org/publicdomain/zero/1.0/) applies to the data made available in this article, unless otherwise stated in a credit line to the data. 


\section{Background}

Heartworm disease, caused by the parasitic nematode Dirofilaria immitis, is a life-threatening disease affecting canines throughout the world [1-5]. Mosquitoes of the Aedes, Anopheles and Culex genera are the primary vectors of D. immitis, which is transmitted when an infected mosquito carrying third-stage larvae (L3) feed on susceptible animals $[1,6]$. The global prevalence of canine heartworm infection varies significantly throughout regions of the world due to a variety of different epidemiological factors, particularly a reliance of mosquito vectors on warm temperatures for survival [1,3]. Within an Australian context, earlier research reported that canine heartworm infection is widespread and highly prevalent, particularly in areas of the states of Queensland (QLD), the Northern Territory and New South Wales (NSW) [5]. The current prevalence of infection in Australia is likely to have significantly reduced in recent years due to the intensive use of macrocyclic lactone (ML) preventatives, yet there is a paucity of prevalence data since the late 1990s [5]. Recent studies have sought to update the prevalence and distribution of $D$. immitis, with the results confirming the endemicity of canine heartworm in QLD [5, 7]. Despite these efforts, information on the occurrence of canine heartworm in reservoir populations, namely rescue and shelter dogs lacking preventative medication, remains scarce.

Diagnosis of canine heartworm infection and disease relies on the detection of $D$. immitis antigen in canine whole blood, serum or plasma and/or detection of $D$. immitis microfilariae (Mff) in whole blood $[4,8,9]$. Currently, enzyme-linked immunosorbent assays (ELISAs) are considered the most effective method of detection of D. immitis antigen [3, 9-11]. Heat treatment of samples prior to $D$. immitis antigen detection assays has been suggested to increase test sensitivity, as it has been shown to free bound antigens from immune complexes $[4,10-$ 15]. However, heat treatment protocols contain multiple steps as well as chemicals to dissociate bound antigens, and have since been excluded from commonly used inhouse test protocols in order to reduce operator error [3, 11, 13, 15-17]. More recently, studies have reported that heat treating samples on a dry heat block prior to antigen detection tends to improve detection of $D$. immitis antigen in a given sample, leading to a higher proportion of positive samples, or to increased optical density (OD) values of positive samples after heat treatment [2, $4,10,17-19]$. Others have proposed a need for immune complex dissociation protocols to be further studied and improved prior to regular heat treatment use $[13,19,20]$.

The aim of this study was to determine the prevalence of heartworm infection as assessed by the presence of canine heartworm antigen or Mff in stray and surrendered shelter dogs in QLD, Australia. Using blood samples collected from these dogs, we evaluated samples before and after heat treatment using a laboratorybased ELISA assay detecting $D$. immitis antigen. Antigen detection was coupled with a modified Knott's test to detect the presence of Mff and with PCR for detection of $D$. immitis DNA. The results of this study enable recommendations for laboratory-based procedures for the detection of $D$. immitis antigen and demonstrate the latitudinal distribution of D. immitis in coastal areas of QLD, Australia.

\section{Methods \\ Study design}

Blood samples $(n=166)$ were collected directly from shelter dogs in QLD, Australia into EDTA blood collection tubes over a period of 3 months (January-March 2020) (Fig. 1). Dogs were housed in one of seven shelter locations (Brisbane, $n=89$; Bundaberg, $n=10$; Cairns, $n=8$; Mackay, $n=11$; Rockhampton, $n=13$; Sunshine Coast, $n=32$; Townsville, $n=3$ ). Blood was collected from all dogs following concent from the shelter owners. Details of each dog (age, gender, source [stray or surrender]) were acquired immediately after blood collection. Blood samples were shipped on ice to the Veterinary Pathology Diagnostic Services (VPDS) laboratory, The University of Sydney, for processing. Whole blood was used immediately for modified Knott's testing. Whole blood and plasma aliquots were stored at $-20{ }^{\circ} \mathrm{C}$ before further processing.

\section{Detection of Mff using the modified Knott's test}

Blood samples were immediately processed using a standard modified Knott's test for the detection of Mff [8]. In brief, blood samples $(1 \mathrm{ml})$ were combined with $2 \%$ buffered formalin $(9 \mathrm{ml})$ in a $15-\mathrm{ml}$ tube and gently homogenised. The homogenate was centrifuged $(10,000 \times g, 10 \mathrm{~min})$ and the supernatant decanted. The resultant pellet was mixed with 2 drops of methylene blue, and enumeration of $D$. immitis Mff was performed under a light microscope at $10 \times$ magnification (Olympus Australia Pty Ltd., Notting Hill, VIC, Australia).

\section{Detection of $D$. immitis antigen before and after heat treatment}

Plasma samples were tested for the presence of $D$. immitis antigen using the DiroCHEK Canine Heartworm Antigen Test Kit (DiroCHEK; Zoetis Australia Pty Ltd., Rhodes, NSW, Australia), which consists of a 96-well format ELISA, according to the manufacturer's instructions. Prior to testing, stored samples were thawed at room temperature and then vortexed for $3 \mathrm{~s}$. Unless sample volume was insufficient, $50 \mu \mathrm{l}$ of unheated plasma was 


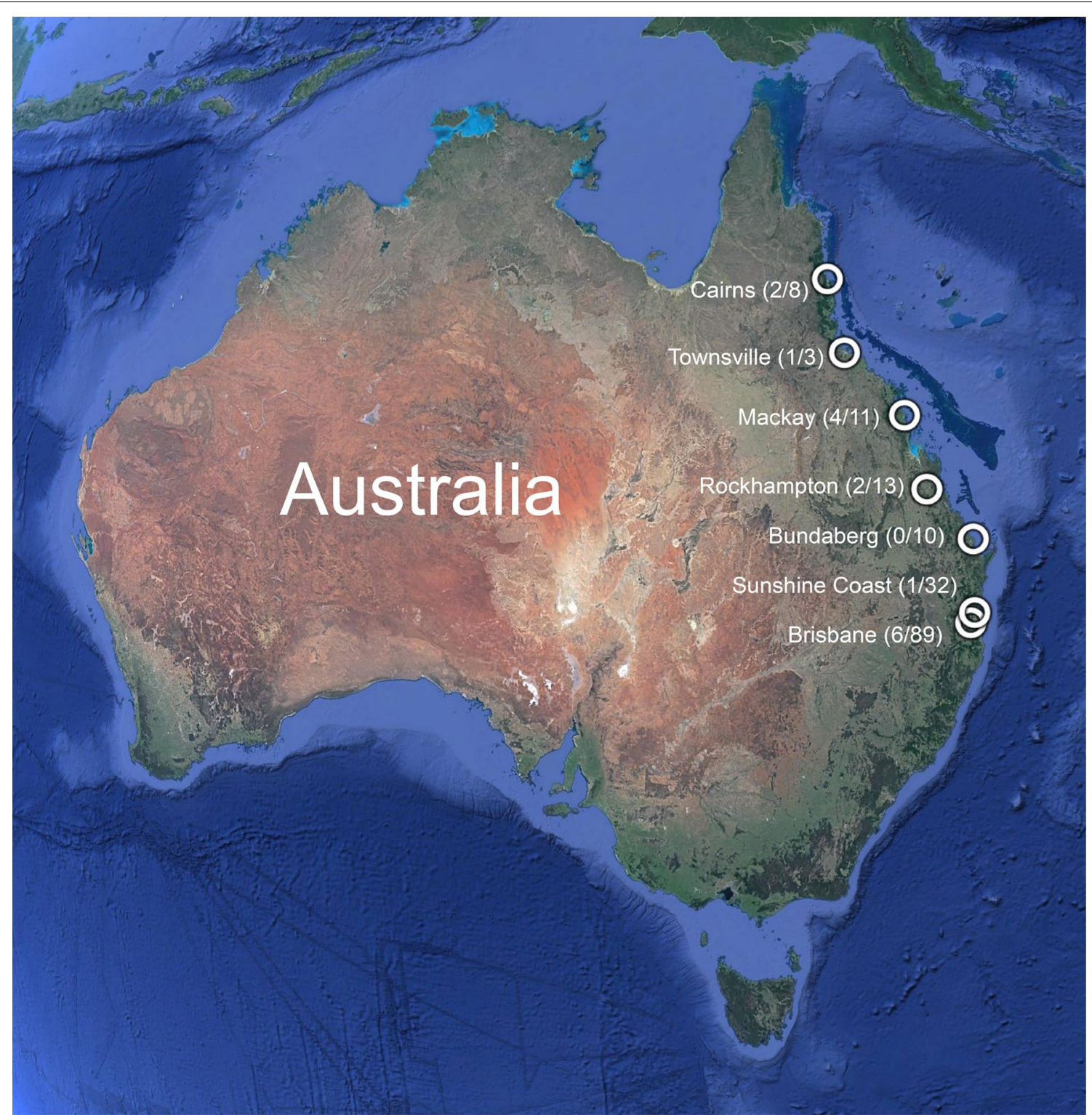

Fig. 1 Location of shelters in Queensland, Australia accessed for the 2020 prevalence surveys where dogs were surveyed for the presence of Dirofilaria immitis antigen. The white circles indicate a location that is associated with the town name, the number in parentheses indicates the number of $D$. immitis-positive dogs using any test/total number of tested dogs. The shortest (airline) distance between Brisbane and Cairns is 1389.40 km. Image from is Google Earth; data are from SIO, NOAA, U.S Navy, NGA, GEBCO (Image Landsat/Copernicus)

tested in duplicate from each sample $(n=165)$. Each testing batch included two positive and two negative control samples provided by the manufacturer.

Heat treatment was performed on plasma samples in duplicate where sufficient volume remained $(n=152)$ according to a previously described method $[10,17,19]$. Briefly, 70-200 $\mu \mathrm{l}$ of plasma was diluted 1:1 with phosphate-buffered saline (PBS; $\mathrm{pH}=7.4$ ) in a 1.5 -ml plain tube and heated to $103^{\circ} \mathrm{C}$ in a dry heat block for $10 \mathrm{~min}$. Samples were then immediately placed on ice for $5 \mathrm{~min}$ before being centrifuged at $>10,000 \times g$ for 5 min using a benchtop centrifuge (Eppendorf Australia, Eppendorf AG, Hamburg, Germany). The supernatant was collected and $50 \mu \mathrm{l}$ was immediately tested using the DiroCHEK ELISA as described above.

A positive result was recorded if the sample developed a visible blue colour after $5 \mathrm{~min}$ of incubation at room temperature, as recommended by the manufacturer (Zoetis Australia). Immediately after samples were visually inspected, each plate was read on an ELISA reader 
(Halo LED96 Microplate Reader; Precisa Gravimetrics AG, Dietikon, Switzerland) using a 620-nm filter, and the OD for each sample was recorded. Samples were considered positive if the OD of the sample exceeded the mean negative control OD by more than three standard deviations.

\section{Molecular detection of $D$. immitis and filarial DNA}

DNA was isolated from $200 \mu \mathrm{l}$ of frozen whole blood samples by following the mammalian blood protocol of the Monarch Genomic DNA Purification Kit (New England Biolabs [Australia] Pty Ltd., Notting Hill, VIC, Australia) and eluted into $100 \mu \mathrm{l}$, before being stored at $-20{ }^{\circ} \mathrm{C}$ prior to processing. The presence of canine DNA was verified using a real-time PCR (qPCR) amplifying the partial canine glyceraldehyde-3-phosphate dehydrogenase (GAPDH; primer set RTPrimerDB ID: 1193), as previously described [5].

For species-specific detection of D. immitis we used a TaqMan probe assay with primers S0624 (F1: 5'-TAG AGG GTC AGC CTG AGT TAT C-3') and S0626 (R1: 5'-AGT AGA ACG TAT ATT CTG AAC AGT AAC C-3') and a TaqMan probe (S0625; 5'-FAM-AGA ACC AAT ACC AAC AGT ATG AAG ACC-BHQ1-3'). Reactions were run at a final volume of $20 \mu \mathrm{l}$, including $2 \mu \mathrm{l}$ template DNA, $10 \mu \mathrm{l}$ of SsoAdvanced Universal Probes Supermix (BioRad Laboratories Pty Ltd., Gladesville, NSW, Australia) and primers and the probe at a final concentration of 400 and $100 \mathrm{nM}$, respectively. The PCR cycling conditions included an initial step at $95^{\circ} \mathrm{C}$, $3 \mathrm{~min}$; followed by 40 two-step cycles of $95{ }^{\circ} \mathrm{C} / 5 \mathrm{~s}$ and $60{ }^{\circ} \mathrm{C} / 15 \mathrm{~s}$. Each run included at least one negative (nontarget control [NTC]) control and a blank DNA isolation control. A positive control DNA sample from a D. immitis adult was included to monitor for PCR inhibition. PCR testing was performed using a CFX96 Touch $^{\mathrm{TM}}$ RealTime PCR Detection System with the corresponding CFX Manager v.3.1 software (BioRad Laboratories Pty Ltd.). The qPCR threshold was determined automatically using default settings and threshold cycle $\left(\mathrm{C}_{\mathrm{q}}\right)$-values were reported for each sample. Samples that amplified with a $\mathrm{C}_{\mathrm{q}}$-value $<40$ were considered positive.

To evaluate the presence of other arthropod-borne Mff, a high-resolution melt (HRM) real-time PCR assay was performed using primers designed by Wongkamchai et al. [21] as previously reported [7]. Positive controls included plasmid DNA for D. immitis and the Acanthocheilonema reconditum 12S rRNA gene (GeneArt; Thermo Fisher Scientific Australia Pty Ltd., Scoresby, VIC, Australia). Each run included at least one negative control. Samples that amplified with a $\mathrm{C}_{\mathrm{q}}$-value $<40$ were sent for DNA sequencing using their respective amplification primers (Macrogen Inc., Seoul, Korea). Sequence chromatographs were manually inspected and compared to a reference $D$. immitis and $A$. reconditium $12 \mathrm{~S}$ rRNA gene sequences using CLC Main Workbench 6.9.1. (CLC Bio; Qiagen Pty Ltd., Chadstone, VIC, Australia).

\section{Statistical analysis}

Samples were categorised into the following risk factors: age ( $\leq 2$ years, $>2$ years old), location (northern QLD [comprising Cairns, Mackay, Rockhampton and Townsville] or southern QLD [comprising Brisbane, Sunshine Coast and Bundaberg]), gender (both entire canine population and neutered males and females, respectively) and source (stray or surrender). Samples were assessed as antigen positive or negative for each of the different risk factors being assessed. Samples were excluded if there were insufficient plasma $(<50 \mu \mathrm{l})$ available to perform the ELISA assay ( $n=1$; one dog from Bundaberg).

Odds ratios (OR) for each of the different risk factors were calculated using binary logistic regression. A forwards stepwise logistic regression model was used to determine the best predictors of sample positivity, and the absence of confounding was verified (IBM SPSS v24; IBM Corp., Armonk, NY, USA). Cohen's kappa coefficient $(\kappa)$ was calculated between replicates to ensure reliability of testing (Microsoft Excel 2010; Microsoft Corp., Redmond, WA, USA). Test power was calculated using 'Post-hoc Power Calculator' (https://clincalc.com/stats).

True prevalence was calculated using previously published specificity and sensitivity values obtained from past studies [9, 22-25]. Sensitivity and specificity values were entered with total counts from this study into Epitools (Ausvet 2020; http://epitools.ausvet.com.au) to calculate true prevalence ranges.

\section{Results \\ Dirofilaria immitis in surrendered and stray dogs from coastal Queensland}

Samples from surrendered and stray dogs from coastal QLD $(n=166)$ were collected for the detection of $D$. immitis antigen. One blood sample had insufficient volume (< $0.5 \mathrm{ml}$; 20.01900-19; Bundaberg) and was excluded for antigen testing. A total of 7.9\% (13/165; 95\% confidence interval [CI]: 4.6-13.1\%) unheated plasma samples tested positive for $D$. immitis antigen according to the DiroCHEK ELISA (Brisbane, 6/89; Sunshine Coast, 1/32; Bundaberg, 0/9; Mackay, 3/11; Rockhampton, 1/13; Townsville, 1/3; Cairns, 1/8). Duplicate testing on unheated plasma samples was performed on $95 / 165$ samples, subject to sample availability (Cohen's $\kappa=0.99$ ).

The modified Knott's test was performed on 161 blood samples (5 samples had insufficient blood volume [ $<1 \mathrm{ml}$; 20.01847-11, 20.01900-9, 20.01900-10, 20.01900-19 and 20-01847-1]). Microfilariae of D. immitis were detected 
in eight blood samples (5.3\%; 8/151; 95\% CI: 2.6-10.3\%; range: $2-3208 \mathrm{Mff} / \mathrm{ml}$ ) from four different shelters (Brisbane, 3/89, Sunshine Coast, 1/32, Cairns, 1/7, Mackay, 3/10).

Canine DNA was successfully isolated from 166 blood samples, confirmed by the detection of canine DNA (average. $\mathrm{C}_{\mathrm{q}}$-value: 20.0). DNA of D. immitis was detected in ten samples using a combination cytochrome $c$ oxidase subunit 1 mitochondrial gene ( $\operatorname{cox} 1)$ and $12 \mathrm{~S}$ rDNA real-time PCR assays (Additional file 1: Table S1). DNA of D. immitis cox 1 was detected using a real-time TaqMan PCR in 8/166 (4.8\%; 95\% CI: 2.3-9.4\%) samples $\left(\mathrm{C}_{\mathrm{q}}\right.$-value range $\left.21.2-30.6\right)$, all of which were Mff-positive according to the modified Knott's test (Additional file 1: Table S1). Using an arthropod-borne HRM realtime PCR assay targeting $12 \mathrm{~S}$ rDNA, all eight Mffpositive samples and a further two amicrofilaraemic samples were confirmed to contain $D$. immitis based on DNA sequences with $100 \%$ homology to reference $12 \mathrm{~S}$ rDNA of $D$. immitis. The two amicrofilaraemic samples (20.01847-8, 20.01900-7; Additional file 1: Table S1) had high $\mathrm{C}_{\mathrm{q}}$-values $(37.3,39.1)$ in the real-time PCR targeting $12 \mathrm{~S}$ rDNA.

\section{Heat treatment increases the OD of the $D$. immitis antigen in positive samples}

Heat treatment was performed on 152 canine plasma samples for which there was sufficient volume, including 54 duplicate samples. In 150/152 samples (Cohen's $\kappa=0.98$ ), heat-treated samples returned identical results to their unheated counterparts. In total, $8.5 \%$ of the submitted samples (14/165; 95\% CI: $5.0-13.8 \%)$ from surrendered and stray dogs from QLD were positive for $D$. immitis antigen using either unheated plasma and/or heat-treated plasma.

Heat treatment did not significantly change the OD of all samples or antigen-negative samples for which both unheated and heat-treated ODs were available (Wilcoxon matched-pairs signed rank test, two-tailed, $P=0.09$ and 0.66 , respectively). Heat treatment, however, significantly increased the OD of the $D$. immitis antigen-positive samples (Wilcoxon matched-pairs signed rank test, twotailed, $P<0.01$ ) (Additional file 1: Table S1). The OD was increased by $>0.1$ for $10 / 14$ (71\%) samples. Two samples had discordant results; the first sample (20.01900-7) was initially negative, but after heat treatment was considered to be antigen positive (Additional file 1: Table S1; note that this sample was PCR positive for D. immitis DNA); the second sample (20.01900-5) was initially considered to be antigen positive, but after heat treatment was considered to be negative and D. immitis DNA was not detected in the PCR analysis (Additional file 1: Table S1).

\section{Location is a significant risk factor for infection with $D$.} immitis

Combining all tests (antigen tests with unheated plasma and heat-treated plasma, modified Knott's tests and PCR) a total of $9.6 \%(16 / 166 ; 95 \%$ CI: $5.9-15.2 \%)$ of samples from surrendered and stray dogs from coastal QLD were positive for D. immitis (Additional file 1: Table S1).

Location was the only risk factor found to be significantly associated with whether a dog tested positive for $D$. immitis antigen $(P=0.01$, binary logistic regression models). No other factors (age, gender or source) were significantly associated with the detection of canine heartworm (Table 1). Sequentially including age, gender and source in the model containing location did not substantially alter the estimated OR, and so none of these factors were considered to be confounders of the relationship between location and odds of testing positive. Dogs located in northern QLD were 4.39-fold (95\% CI: 1.2613.51) more likely to test $D$. immitis antigen positive than dogs located in southern QLD (Table 1). Including all $D$. immitis antigen- and/or Mff-positive samples (modified Knott's test, PCR; see Additional file 1: Table S1) had no effect on the above result (OR: 6.13; 95\% CI: 2.09-17.86). The tests probably had low power, however, for age $(3.7 \%$ post-hoc power) and source (22\% post-hoc power).

Combining the results of the DiroCHEK ELISA on unheated and heat-treated plasma, samples collected from northern QLD revealed an apparent $D$. immitis prevalence of $20.0 \%$ (7 positive samples/35 tested) and an estimated true prevalence in the range of $8.0-29.1 \%$, which was significantly higher than that of southern QLD where samples revealed an apparent $D$. immitis prevalence of $5.4 \%$ (7 positive/130 tested) and an estimated true prevalence in the range of $0.0-7.0 \%$ (Tables 1,2 ).

\section{Discussion}

Location is a significant risk factor for canine heartworm infection

Our study demonstrate that the location of the animal (northern or southern QLD) poses a significant risk for the detection of $D$. immitis antigen in surrendered and stray dogs in coastal Queensland, Australia (Table 1). Early research has recognised that differences in the distribution of canine heartworm in Australia are likely due to the distribution of the multiple vector species $[26,27]$. Mosquitoes of the Aedes, Culex and Anopheles genera have been demonstrated as the main vectors within Australia [28-31]. In order for transmission between the vector and the host to occur, the mosquito becomes infected through feeding on a microfilaraemic host, following which $D$. immitis matures from first-stage larva (L1) to L3 within the mosquito [3]. This 
Table 1 Risk factors in dogs tested for Dirofilaria immitis antigen from Queensland, Australia

\begin{tabular}{|c|c|c|c|c|c|c|}
\hline Risk factors & $\begin{array}{l}\text { Positive for D. immitis } \\
\text { antigen }^{\text {a }}\end{array}$ & $\begin{array}{l}\text { Negative for D. immitis } \\
\text { antigen }\end{array}$ & Total & $P$ value & Odds ratio & $\begin{array}{l}95 \% \\
\text { Confidence } \\
\text { interval }\end{array}$ \\
\hline Location $^{\text {b }}$ & & & & 0.010 & & \\
\hline Southern QLD & 7 & 123 & 130 & & 1 & - \\
\hline Northern QLD & 7 & 28 & 35 & & 4.39 & $1.26-13.51$ \\
\hline Sex/status & & & & 0.474 & & \\
\hline Male neutered & 3 & 64 & 67 & & 1 & - \\
\hline Female entire & 4 & 25 & 29 & & 3.41 & $0.71-16.36$ \\
\hline Female neutered & 5 & 43 & 48 & & 2.48 & $0.56-10.93$ \\
\hline Male entire & 2 & 19 & 21 & & 2.25 & $0.35-14.44$ \\
\hline Age & & & & 0.868 & & \\
\hline$\leq 2$ years & 7 & 72 & 79 & & 1 & - \\
\hline$>2$ years & 7 & 79 & 86 & & 0.91 & $0.31-2.73$ \\
\hline Source & & & & 0.299 & & \\
\hline Stray & 10 & 125 & 135 & & 1 & - \\
\hline Surrender & 4 & 26 & 30 & & 1.92 & $0.56-6.61$ \\
\hline Total & 14 & 151 & 165 & & & \\
\hline
\end{tabular}

${ }^{a}$ Positive and negative counts are based on visual assessment of combined results for unheated and heat-treated plasma samples

${ }^{b}$ Northern Queensland (QLD) consists of samples obtained from Cairns, Mackay, Rockhampton and Townsville; and southern QLD consists of samples from Brisbane, Sunshine Coast and Bundaberg

Table 2 Apparent and true prevalence for canine heartworm antigen positivity of dogs from northern and southern Queensland, Australia

\begin{tabular}{lll}
\hline Location & $\begin{array}{l}\text { Apparent prevalence }(95 \% \\
\text { confidience interval, } n)\end{array}$ & True prevalence \\
\hline Northern QLD & $20.0 \%(9.7-36.2 \%, 35)$ & $8.0-29.1 \%$ \\
Southern QLD & $5.4 \%(2.4-10.9 \%, 130)$ & $0.0-7.0 \%$ \\
Overall QLD & $8.5 \%(5.0-13.8 \%, 165)$ & $0.0-11.0 \%$ \\
\hline
\end{tabular}

The test results are based on combined unheated and heat-treated plasma testing. Sensitivity and specificity values used were chosen from previously published reports as follows: $0.88,0.95 ; 0.77,0.85 ; 0.90,0.96 ; 0.86,0.97 ; 0.77,1$; $0.85,1 ; 0.71,0.94 ; 0.63,0.97[9,22-25]$

maturation process is temperature dependent, with ideal conditions of $27{ }^{\circ} \mathrm{C}$ with $80 \%$ humidity allowing development within 10-14 days. The development process is more rapid under warmer conditions and slower under cooler conditions [3, 32, 33].

Multiple studies have demonstrated the influence of climate and climate change on the distribution of mosquito vectors, and thus the occurrence of canine heartworm infection [1, 7, 27, 32-35]. These implications are relevant to the current study as the average minimummaximum temperature and humidity between 1999 and 2020 in Brisbane (southern QLD) was $16.4-26.6{ }^{\circ} \mathrm{C}$ and $52-63 \%$, compared to an average temperature of 20.8-29.1 ${ }^{\circ} \mathrm{C}$ and $62-72 \%$ humidity in Cairns (northern QLD) [36, 37]. Climatic differences across QLD suggest that these variations are responsible for an increased number of mosquito vectors in northern QLD, and for a decreased maturation time of $D$. immitis within mosquitoes. Our results found that dogs residing in northern QLD are at greater risk of developing canine heartworm disease than dogs residing in southern QLD, which is supported by climatic data [36,37]. Further research is needed in this area to gain an accurate idea of the distribution of mosquito vectors across different regions of QLD, Australia.

\section{Prevalence of canine heartworm infection in Queensland, Australia}

Canine heartworm infection has been reported in QLD with a prevalence between 11.8 and 36\% [27, 38, 39]. These reports are largely out of date and are unlikely to reflect the current prevalence due to the uptake of heartworm preventatives in Australia [5, 40]. More recently, a 2016 paper confirmed the endemicity of canine heartworm in coastal areas of central QLD, Australia, despite a majority of the cohort being treated with monthly heartworm preventatives [7]. The current study demonstrates the prevalence of D. immitis antigen in 20.0 and $5.4 \%$ of surrendered and stray dogs from northern and southern QLD, respectively (Table 2). The ELISA antigen test used in this study performs well in detecting $D$. immitis infection in dogs with female worms, but it is well acknowledged that the sensitivity is lowered for infections from a single female or male-only infections [9, 22-25]. In a 
low burden cohort study of necropsy-confirmed positive dogs from the USA, the sensitivity and specificity of DiroCHEK ELISA was 94\% (95\% CI: 76-98\%) and 94\% (95\% CI: 98-97\%), respectively, for dogs with at least two adult female D. immitis [9]. In the same study, using all infected animals regardless of the burden, the sensitivity was 71\% (95\% CI: 62-79\%) [9]. Similarly, the sensitivity and specificity of the DiroCHEK ELISA was 62.5 and $97.4 \%$, respectively, using necropsy-confirmed positive dogs from Australia [23]. As our results are lower than historical prevalence estimates, they confirm the expectation that prevalence of the disease has decreased with the introduction of ML heartworm preventative medications $[7,26,38,39]$. A limitation of this study is the incomplete or unknown history of canine heartworm prevention in the dogs tested herein, and hence the reported prevalence is potentially an underestimation of the true extent of D. immitis in QLD, Australia.

Donnett et al. [41] hypothesised that there was likely to be an increased prevalence of canine heartworm infection in shelter dogs compared to privately owned dogs due to increased preventative administration in the latter group. Based on the results of their study, these authors concluded that the presence of $D$. immitis was $34.4 \%$ higher in shelter dogs than owned dogs in Mississippi, USA, and suggested that these dogs could serve as reservoirs of heartworm infection for the rest of the country [41]. Similarly, in 2010 Tzipory et al. [42] found a significantly higher prevalence of $D$. immitis infections in shelter dogs $(14.6 \%)$ compared to pet dogs $(<2 \%)$ [42]. Clearly, heartworm is still endemic in QLD, but the true prevalence in the owned dog population remains unknown, and implications of a high $D$. immitis prevalence within QLD shelter dogs on the wider owned dog population are also unknown. Future research is required to determine the number and distribution of owned dogs in QLD affected by canine heartworm infection, and to estimate the overall $D$. immitis prevalence.

Our results indicate that heat treatment did not have a significant effect on the detection of $D$. immitis antigen within canine plasma samples collected in QLD, Australia. In the past, studies explored the role of heat treatment in freeing $D$. immitis antigen, which is bound or trapped within immune complexes within canine plasma. Antigens freed from immune complexes resulted in an increased sensitivity of detection of $D$. immitis antigen post-heat treatment $[2,4,10,13,17,19,43-45]$. This theory of heat-induced immune complex destruction is based on earlier literature $[11,16]$. Unfortunately, the protocols described in these studies are complex and require multiple steps and solutions. Further, Weil et al. [11] states that the success of their proposed assay was only in part due to pre-treatment with heat [11].
Therefore, findings in the recent literature about the impact of heat-treatment on assay sensitivity should be interpreted with caution. The American Heartworm Society (https://www.heartwormsociety.org/) [3] does not currently recommend heat treatment in conjunction with in-house antigen testing as it is unknown whether there may be cross-reactivity with other helminth antigens due to the heating of plasma samples. Antigen testing is now done with whole blood, sera and plasma, and it appears to be unknown if the heat treatment effect would be consistent across all three sample types. Instead, it is recommended that Mff testing be performed in combination with antigen testing [3]. The heat-treatment prior to $D$. immitis antigen testing may not be recommended for all dogs but may be beneficial for certain types of patients, as summarised in Table 5 in Little et al. [14]. Studies analysing known $D$. immitis-positive dogs, including exact worm burden and co-infection, will provide the needed insight into the value of heat treatment prior to testing for D. immitis antigen $[13,46]$.

The modified Knott's test is traditionally used to detect Mff and, when coupled with microscopy, D. immitis can be reliably identified $[8,47,48]$. It is known, however, that a large proportion of heartworm-infected dogs are amicrofilaraemic [26]. For example, in QLD, Australia, Atwell et al. [49] detected Mff using the modified Knott's test in $15 / 26$ dogs confirmed to have patent infection at necropsy (35\% amicrofilaraemic dogs) [49]. The DiroCHEK ELISA successfully identified 8/15 amicrofilaraemic dogs as $D$. immitis positive and the sensitivity for amicrofilaraemic dogs was estimated to be $53.3 \%$ (95\% CI: $25.1-$ 74.8\%) [49]. An alternative approach to the concentration and visualisation of Mff using the modified Knott's test is detecting $D$. immitis DNA in the blood samples.

There are several molecular tests that are either $D$. immitis specific or amplify conserved regions across other filarial nematodes $[21,50]$. In this study, we showed that PCR reliably detected $D$. immitis in all Mff-positive samples, including those with as few as $2 \mathrm{Mff} / \mathrm{ml}$. For PCR, we used only $0.1 \mathrm{ml}$ of blood compared to the $1 \mathrm{ml}$ required to conduct the modified Knott's test demonstrating the usefulness of the former approach in lowvolume samples. Successful amplification of samples with $2 \mathrm{Mff} / \mathrm{ml}$ is valuable because it suggests the presence of cell-free DNA (not confined to Mff) and that PCR can be used in low microfilaraemic samples $(<10 \mathrm{Mff} / \mathrm{ml})$.

\section{Conclusions}

In this study we confirmed the current presence of $D$. immitis in shelter dogs along the coastal region of QLD, Australia. Our results demonstrate that dogs located in northern QLD are at significantly higher risk of being $D$. immitis positive than dogs located in southern QLD. Heat 
treatment of canine plasma demonstrated no significant additional effect on whether samples were classified as $D$. immitis antigen positive or negative using the DiroCHEK ELISA. A combination of D. immitis antigen testing with the detection of Mff either using the modified Knott's test or PCR is preferred to maximise detection of D. immitispositive dogs.

\section{Abbreviations}

ELISA: Enzyme-linked immunosorbent assay; Mff: Microfilariae; ML: Macrocyclic lactone; NSW: New South Wales; PBS: Phosphate-buffered saline; QLD: Queensland.

\section{Supplementary Information}

The online version contains supplementary material available at https://doi. org/10.1186/s13071-021-04896-y.

Additional file 1: Table S1. Summary of Dirofilaria immitis-positive diagnostics results for Queensland, Australia dogs from shelters.

\section{Acknowledgements}

We acknowledge the technical and logistical support provided by the Veterinary Pathology Diagnostics Services (VPDS) at The University of Sydney, RSPCA QLD, Rockhampton Council and Bundaberg Council. This work was completed in partial fulfillment for the requirements of the Doctor of Veterinary Medicine degree, The University of Sydney (JP). We thank John Messer, Tinh-Son Nguyen, Ilze Nel and Richard L'Estrange (Zoetis Australia Pty Ltd), and Andy Hodge (Zoetis Australia Research and Manufacturing Pty Ltd) for comments on the earlier versions of this manuscript.

\section{Authors' contributions}

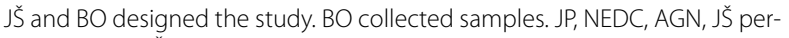
formed tests. JS and MPW supervised laboratory work and statistical analysis.

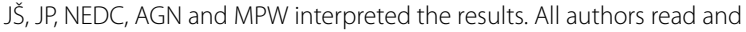
approved the final manuscript.

\section{Funding}

This work was supported by Zoetis Australia Pty Ltd. BO is supported by an Australian Government Research Training Program scholarship.

\section{Availability of data and materials}

The raw data analysed in this article is available at LabArchives: http://dx.doi. org/10.25833/kvb5-2s19.

\section{Declarations}

\section{Ethics approval and consent to participate}

Animal ethics (2019/1619) approval was obtained from The University of Sydney's Animal Ethics Committee.

\section{Consent for publication}

All authors have approved the manuscript for submission.

\section{Competing interests}

JŠ received funds from Zoetis Australia Pty Ltd. The authors declare that they have no further competing interests.

Received: 14 May 2021 Accepted: 24 July 2021

Published online: 09 August 2021

\section{References}

1. Simon F, Siles-Lucas M, Morchon R, Gonzalez-Miguel J, Mellado I, Carreton $E$, et al. Human and animal dirofilariasis: the emergence of a zoonotic mosaic. Clin Microbiol Rev. 2012;25:507-44.

2. Starkey LA, Bowles JV, Payton ME, Blagburn BL. Comparative evaluation of commercially available point-of-care heartworm antigen tests using well-characterized canine plasma samples. Parasites Vectors. 2017;10:475.

3. Nelson CT, McCall JW, Jones S, Moorhead A. Current canine guidelines for the prevention, diagnosis and management of heartworm (Dirofilaria immitis) infection in dogs. Wilmington: American Heartworm Society; 2018.

4. Panarese R, latta R, Mendoza-Roldan JA, Szlosek D, Braff J, Liu J, et al. Comparison of diagnostic tools for the detection of Dirofilaria immitis infection in dogs. Pathogens. 2020;9:499.

5. Orr B, Ma G, Koh WL, Malik R, Norris JM, Westman ME, et al. Pig-hunting dogs are an at-risk population for canine heartworm (Dirofilaria immitis) infection in eastern Australia. Parasites Vectors. 2020;13:69.

6. Bowman DD, Atkins CE. Heartworm biology, treatment, and control. Vet Clin North Am Small Anim Pract. 2009;39:1127-58.

7. Nguyen C, Koh WL, Casteriano A, Beijerink N, Godfrey C, Brown G, et al. Mosquito-borne heartworm Dirofilaria immitis in dogs from Australia. Parasites Vectors. 2016;9:535.

8. Kelly JD. Detection and differentiation of microfilariae in canine blood. Aust Vet J. 1973;49:23-7.

9. Courtney $\mathrm{CH}$, Zeng QY. Comparison of heartworm antigen test kit performance in dogs having low heartworm burdens. Vet Parasitol. 2001:96:317-22.

10. Velasquez L, Blagburn BL, Duncan-Decoq R, Johnson EM, Allen KE, Meinkoth J, et al. Increased prevalence of Dirofilaria immitis antigen in canine samples after heat treatment. Vet Parasitol. 2014;206:67-70.

11. Weil GJ, Malane MS, Powers KG, Blair LS. Monocloncal antibodies to parasite antigens found in the serum of Dirofilaria immitis-infected dogs. $J$ Immunol. 1985;134(2):1185-91.

12. Wang D, Bowman DD, Brown HE, Harrington LC, Kaufman PE, McKay T, et al. Factors influencing U.S. canine heartworm (Dirofilaria immitis) prevalence. Parasites Vectors. 2014;7:264.

13. Gruntmeir JM, Long MT, Blagburn BL, Walden HS. Canine heartworm and heat treatment: an evaluation using a well based enzyme-linked immunosorbent assay (ELISA) and canine sera with confirmed heartworm infection status. Vet Parasitol. 2020;283:109169.

14. Little S, Saleh M, Wohltjen M, Nagamori Y. Prime detection of Dirofilaria immitis: understanding the influence of blocked antigen on heartworm test performance. Parasites Vectors. 2018;11:186.

15. Weil GJ, Blair LS, Ewanciw DV, Malatesta PF. Use of parasite antigen detection to monitor the success of drug therapy in Dirofilaria immitis-infected dogs. J Parasitol. 1986;72:737-40.

16. Weil GJ, Malane MS, Powers KG. Detection of circulating parasite antigens in canine dirofilariasis by counterimmunoelectrophoresis. Am J Trop Med Hyg. 1984;33:425-30.

17. Little SE, Munzing C, Heise SR, Allen KE, Starkey LA, Johnson EM, et al. Pretreatment with heat facilitates detection of antigen of Dirofilaria immitis in canine samples. Vet Parasitol. 2014;203:250-2.

18. Drake J, Gruntmeir J, Merritt H, Allen L, Little SE. False negative antigen tests in dogs infected with heartworm and placed on macrocyclic lactone preventives. Parasites Vectors. 2015:8:68.

19. DiGangi BA, Dworkin C, Stull JW, O'Quin J, Elser M, Marsh AE, et al. Impact of heat treatment on Dirofilaria immitis antigen detection in shelter dogs. Parasites Vectors. 2017;10(Suppl 2):483.

20. Savadelis MD, Roveto JL, Ohmes CM, Hostetler JA, Settje TL, Dzimianski MT, et al. Evaluation of heat-treating heartworm-positive canine serum samples during treatment with Advantage Multi ${ }^{\circledR}$ for Dogs and doxycycline. Parasites Vectors. 2018;11:98.

21. Wongkamchai S, Monkong N, Mahannol P, Taweethavonsawat P, Loymak S, Foongladda S. Rapid detection and identification of Brugia malayi, $B$. pahangi, and Dirofilaria immitis by high-resolution melting assay. Vector Borne Zoonotic Dis. 2013;13:31-6.

22. Rohrbach BW, Patton S. Effects of diagnostic test accuracy and treatment efficacy on the occurrence of suspected failure of heartworm prophylaxis in dogs. J Vet Intern Med. 2013;27:791-7.

23. Collins GH, Pope SE. An evaluation of an ELISA test for the detection of antigens of Dirofilaria immitis. Aust Vet J. 1987;64:318-9. 
24. Courtney $\mathrm{CH}$, Zeng MD. Sensitivity and specificity of two heartworm antigen tests. Canine Pract. 1993;18:20-2.

25. Hoover JP, Campbell GA, Fox JC, Claypool PL, Mullins SB. Comparison of eight diagnostic blood tests for heartworm infection in dogs. Canine Pract. 1996;21:11-9.

26. Bidgood A, Collins GH. The prevalence of Dirofilaria immitis in dogs in Sydney. Aust Vet J. 1996;73:103-4.

27. Welch JS, Dobson C, Freeman C. Distribution and diagnosis of dirofilariasis and toxocariasis in Australia. Aust Vet J. 1979:55:265-74.

28. Bemrick WJ, Moorhouse DE. Potential vectors of Dirofilaria immitis in the Brisbane area of Queensland, Australia. J Med Entomol. 1968;5:269-72.

29. Russell RC, Geary MJ. The influence of microfilarial density of dog heartworm Dirofilaria immitis on infection rate and survival of Aedes notoscriptus and Culex annulirostris from Australia. Med Vet Entomol. 1996;10:29-34.

30. Russell RC. The relative importance of various mosquitoes for the transmission and control of dog heartworm in south-eastern Australia. Aust Vet J. 1990;67:191-2.

31. Russell RC, Geary MJ. The susceptibility of the mosquitoes Aedes notoscriptus and Culex annulirostris to infection with dog heartworm Dirofilaria immitis and their vector efficiency. Med Vet Entomol. 1992;6:154-8.

32. Dearsley EJ, O'Handley RM, Caraguel CGB. Is canine heartworm (Dirofilaria immitis) endemic to South Australia? Aust Vet J. 2019;97:191-6.

33. Sassnau R, Daugschies A, Lendner M, Genchi C. Climate suitability for the transmission of Dirofilaria immitis and D. repens in Germany. Vet Parasitol. 2014;205:239-45.

34. Montoya-Alonso JA, Carreton E, Morchon R, Silveira-Viera L, Falcon $Y$, Simon F. The impact of the climate on the epidemiology of Dirofilaria immitis in the pet population of the Canary Islands. Vet Parasitol. 2016;216:66-71.

35. Genchi C, Rinaldi L, Cascone C, Mortarino M, Cringoli G. Is heartworm disease really spreading in Europe? Vet Parasitol. 2005;133:137-48.

36. Bureau of Meteorology (BOM). Climate statistics for Australian locations: Brisbane. 2020. Australian Government, Canberra. http://www.bom.gov. au/climate/averages/tables/cw_040214.shtml. Accessed 24 Aug 2020.

37. Bureau of Meteorology (BOM). Climate statistics for Australian locations: Cairns. 2020. Australian Government, Canberra. http://www.bom.gov.au/ climate/averages/tables/cw_031011.shtml. Accessed 24 Aug 2020.

38. Carlisle $\mathrm{CH}$, Atwell RB. A survey of heartworm in dogs in Australia. Aust Vet J. 1984;61:356-60.
39. Atwell RB, Carlisle C. Canine dirofilariasis in the metropolitan area of Brisbane. Aust Vet J. 1979;55:399.

40. Doyle K. Heartworm treatment—NRA decision opposed. Aust Vet J. 2001;79:74.

41. Donnett U, Hubbard K, Woodruff K, Varela-Stokes A. Prevalence of canine heartworm infection in Mississippi animal shelters. Vet Parasitol. 2018;259:68-73.

42. Tzipory N, Crawford PC, Levy JK. Prevalence of Dirofilaria immitis, Ehrlichia canis, and Borrelia burgdorferi in pet dogs, racing greyhounds, and shelter dogs in Florida. Vet Parasitol. 2010;171:136-9.

43. Carmichael J, McCall S, DiCosty U, Mansour A, Roycroft L. Evaluation of Dirofilaria immitis antigen detection comparing heated and unheated serum in dogs with experimental heartworm infections. Parasites Vectors. 2017;10(Suppl 2):486.

44. Little SE, Raymond MR, Thomas JE, Gruntmeir J, Hostetler JA, Meinkoth $\mathrm{JH}$, et al. Heat treatment prior to testing allows detection of antigen of Dirofilaria immitis in feline serum. Parasites Vectors. 2014;7:1.

45. Beall MJ, Arguello-Marin A, Drexel J, Liu J, Chandrashekar R, Alleman AR. Validation of immune complex dissociation methods for use with heartworm antigen tests. Parasites Vectors. 2017:10:115-235.

46. Gruntmeir JM, Thompson NM, Long MT, Blagburn BL, Walden HDS. Detection of heartworm antigen without cross-reactivity to helminths and protozoa following heat treatment of canine serum. Parasites Vectors. $2021 ; 14: 71$.

47. Newton WL, Wright WH. The occurrence of a dog filariid other than Dirofilaria immitis in the United States. J Parasitol. 1956;42:246-58.

48. Mylonakis ME, Papadopoulos E, Koutinas AF, Paitaki C, Leontides L. Comparative methodology for the detection and differentiation of circulating microfilariae of Dirofilaria immitis in the dog. J Helminthol. 2004;78:137-40.

49. Atwell RB, Sheridan AB, Baldock FC. An evaluation of the Dirochek test for detection of Dirofilaria immitis antigen in dogs. Aust Vet J. 1988;65:161-2.

50. Rishniw M, Barr SC, Simpson KW, Frongillo MF, Franz M, Dominguez Alpizar JL. Discrimination between six species of canine microfilariae by a single polymerase chain reaction. Vet Parasitol. 2006;135:303-14.

\section{Publisher's Note}

Springer Nature remains neutral with regard to jurisdictional claims in published maps and institutional affiliations.
Ready to submit your research? Choose BMC and benefit from:

- fast, convenient online submission

- thorough peer review by experienced researchers in your field

- rapid publication on acceptance

- support for research data, including large and complex data types

- gold Open Access which fosters wider collaboration and increased citations

- maximum visibility for your research: over $100 \mathrm{M}$ website views per year

At BMC, research is always in progress.

Learn more biomedcentral.com/submissions 